\title{
Quantitative texture analysis of polyphased oxides by diffraction: example of Bi2223 sinter-forged ceramic and Y123 foam superconductors
}

\author{
E. Guilmeau $^{\text {a,* }}$, S. Lambert ${ }^{\text {a }}$, D. Chateigner ${ }^{\text {a }}$, J.G. Noudem ${ }^{\text {a }}$, B. Ouladiaf ${ }^{b}$ \\ ${ }^{a}$ CRISMAT-ENSICAEN, 6 Bd. Marechal Juin, 14050 Caen, France \\ ${ }^{\mathrm{b}}$ ILL, BP 156, 38042 Grenoble, France
}

\begin{abstract}
This paper deals about the texture analysis of sinter-forging $(\mathrm{Bi}, \mathrm{Pb})_{2} \mathrm{Sr}_{2} \mathrm{Ca}_{2} \mathrm{Cu}_{3} \mathrm{O}_{10+\delta}(\mathrm{Bi} 2223)$ discs and $\mathrm{YBa}_{2} \mathrm{Cu}_{3} \mathrm{O}_{7-x}\left(\mathrm{Y}_{123}\right)$ foams. Orientation distributions were measured by X-ray and neutron diffraction respectively. In the case of Bi2223 samples, an overview of our results is presented. It reflects clearly the importance to combine physical measurements and texture analysis. Several factors as the size of the precursor powder and the process parameters influence significantly the crystallite orientation and consequently the final properties of the textured discs. In the case of Y123 foams, the only usable non destructive diffraction characterisation is the combined approach, and we show what it can bring on one example.

(C) 2003 Elsevier B.V. All rights reserved.
\end{abstract}

Keywords: Bi2223 phase; Y123; Foams; Texture

\section{Introduction}

The development of texture in polycrystalline materials is a topic of recent interest in ceramics processing, since it allows an optimisation of physical properties. More particularly, the Bi2223 and Y123 superconductor compounds require a precise control of the texture. Their high structural anisotropy results in larger superconducting current properties in $(\mathrm{ab})$ planes than along the $c$-axis. As a result of this anisotropy, it appears necessary to induce a preferential orientation of $c$-axis during the elaboration. This $(00 \ell)$ texture will favour the circulation of supercurrents along (ab) planes and a great improvement of critical current densities $\left(J_{\mathrm{c}}\right)$. However, correlating changes in texture development with processing parameters is a complex matter. Using the simplest techniques such as measuring $\theta-2 \theta$ diagrams, the effect of texture can only be measured qualitatively, and the results concern only planes perpendicular to the incident plane of rays. The comparison of relative peak intensity, widely used in the Lotgering factor method [1] for instance, does not provide information on the orientation distribution of crystals (OD), which can be the key in understanding texture-phase-structure-processing relationships. If this

\footnotetext{
* Corresponding author.

E-mail address: emmanuel.guilmeau@ismra.fr (E. Guilmeau).
}

factor is somehow linked to the strength of the texture, this link is not physically interpretable. The rocking curve strategy may be aimed to quantitatively measure the texture, provided another axis of rotation is added ( $\Psi$ for example). However, this technique is suitable for very low angle ranges (typically less than $5^{\circ}$ of misorientation), then is limited to single crystals or epitaxial layers, due to absorption limitations [2]. In our case, orientation dispersions of more than $10^{\circ}$ have to be characterised, and we are interested in the distribution of all crystallites in a quantitative way. The most appropriate method for such quantitative texture determination is the calculation of the OD from pole figure measurement [3], which we use here. For textured Bi2223 discs, it is then possible to extract the distribution of crystallites orientations around $c$-axis [4-6], and for Y123 foams using neutron experiment, the distribution of orientations in the whole volume of irregularly shaped samples.

\section{Experimental procedure}

We used X-ray and neutron diffraction for the quantitative texture analysis of our samples, depending on their shape, crystallite sizes and homogeneity. X-ray investigation are preferred for regular shapes and homogeneous samples with small crystallites (compared to the beam size), while 
probing the whole volume at the centimetre cube size is reserved to neutron probing. We used experimental strategies detailed elsewhere both using neutrons [7] and X-rays [8]. The key point in our measurements is the presence of several low crystal-symmetry phases which obliges us either to restrict the measured angular range, when possible [4], or to use position sensitive detectors in order to avoid prohibited acquisition times [8]. The important input of such procedure is the availability to calculate afterwards phase ratios, orientation distribution functions (ODFs), structures, crystallite sizes and microstrains, residual stresses..., which takes the name of combined analysis [9].

\section{Crystallite orientation distributions in Bi2223 textured dises}

Bi2223 discs were textured by the sinter-forging method as described elsewhere [10]. Electrical properties were measured at $77 \mathrm{~K}$ and self-field by standard four-point contacts method, applying a $\mu \mathrm{V} \mathrm{cm} \mathrm{cm}^{-1}$ criteria for the critical current.

A first interesting result concerns the influence of particle size distributions on the critical current densities. In Fig. 1, the full width at half maximum of the OD (FWHD) of the Bi2212 and Bi2223 phases are compared to the critical current density versus the sieve size used to obtain three different powders. The critical current density decreases from $7000 \mathrm{~A} \mathrm{~cm}^{-2}$ for a sieve size equal to $63 \mu \mathrm{m}$ to $4000 \mathrm{~A}$ $\mathrm{cm}^{-2}$ for a sieve size equal to $200 \mu \mathrm{m}$. From this figure, it becomes evident that larger particles induce worse grain alignments of both phases, particularly when these particles are composed of many smaller, randomly oriented grains, and poor connectivity, which explains the weak transport critical current density of sample composed of powder obtained from a $200 \mu \mathrm{m}$ sieve. The Bi2212 plate-like grains present in the agglomerates move with difficulty and lead to more dispersed textures characterized by lower critical current densities. Furthermore, the large agglomerates result in the existence of large residual phases which hinder somehow the grain alignment of the Bi2223 grains in the matrices and act as barriers for the current paths. The resulting transport properties are, of course, deteriorated.

The difference in FWHDs observed between the two major phases also increases with the sieve size, with the FWHD of Bi2223 constantly smaller than that of Bi2212. This is a strong indication for the initial nucleation of Bi2223 on Bi2212 particles, followed by the naturally anisotropic growth of Bi2223 crystals. When only small and well-oriented Bi2212 particles exist, they all independently serve as nucleation sites for Bi2223, and the Bi2212 and Bi2223 phases exhibit identical FWHDs. On the contrary, in the presence of large agglomerates, Bi2223 nucleates on the Bi2212 crystallites, both on the surface and inside the agglomerates. In both cases, Bi2223 crystallites are able to grow and rotate under the uniaxial pressure as they develop, hereby gaining in texture strength, but with a less degree when Bi2223 forms from the misaligned Bi2212 crystals on the inside of the agglomerates.

A second promising result is the influence of sinter-forging time applied during the process versus Bi2223 content, $J_{\mathrm{c}}$ and FWHD values. The sinter-forging temperature was fixed at $845^{\circ} \mathrm{C}$. On Fig. 2, it is clearly shown that the Bi2223 phase content and $J_{\mathrm{c}}$ increase as the sintering time increases. At the same time, we observe that the FWHD

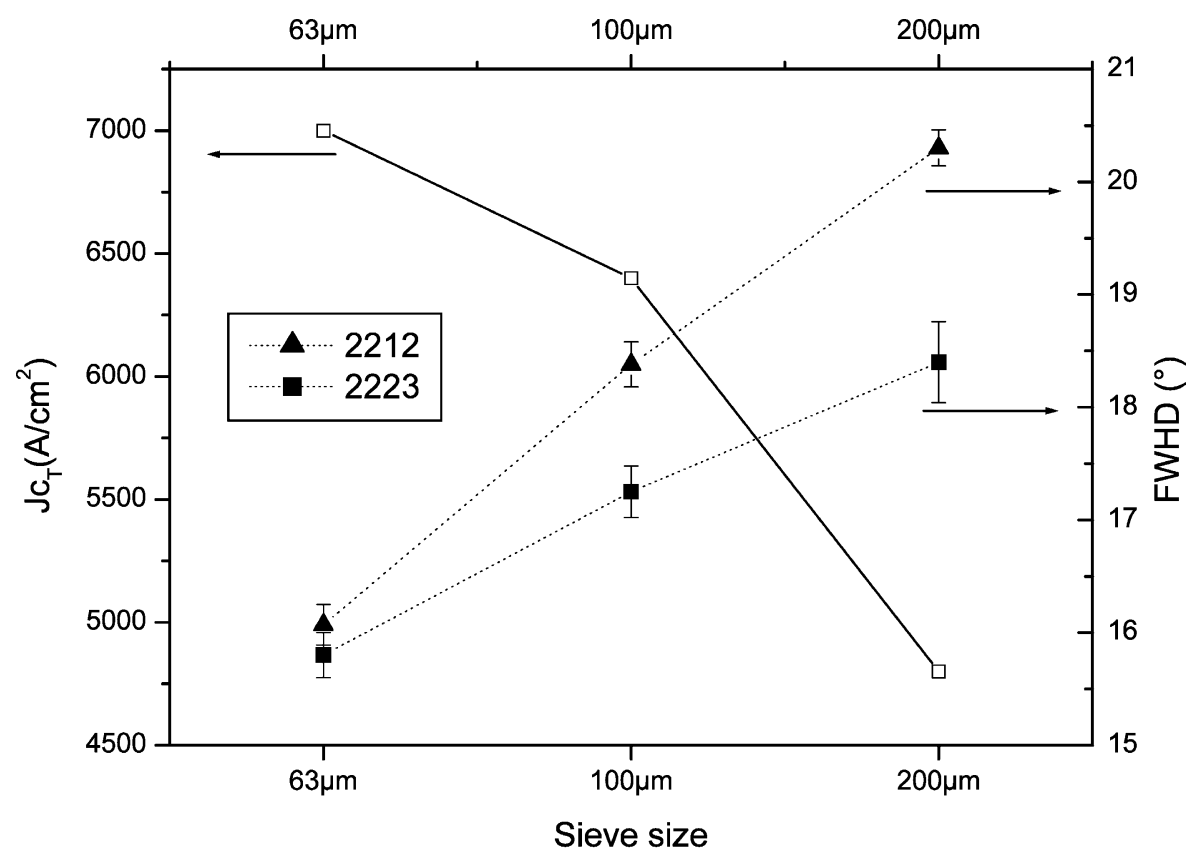

Fig. 1. Comparison of transport critical current density and FWHD with sieve size [4]. 


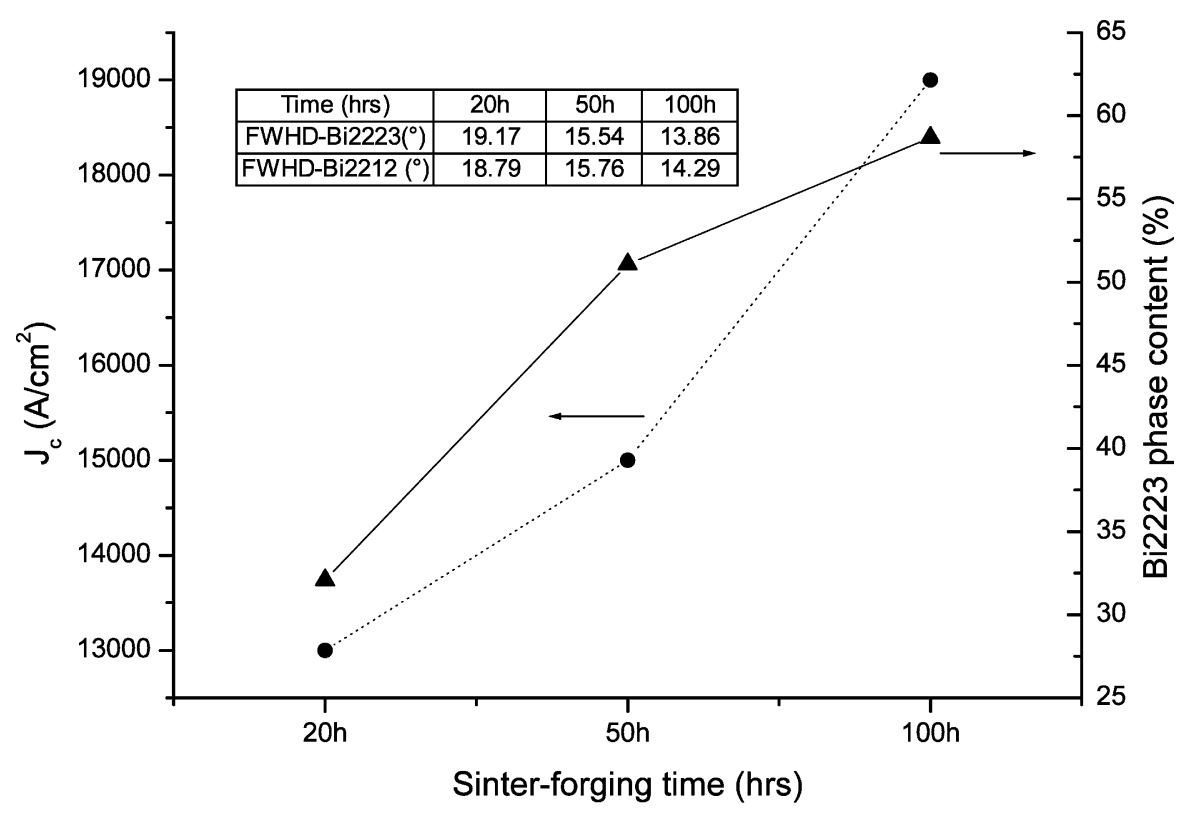

Fig. 2. Bi2223 phase content and transport critical current density versus sinter-forging time. Inset showing the corresponding crystallite ODs of the Bi2212 and Bi2223 phases [5].

values decrease. It is of course easy to compare the trend of each factor. The improvement of $J_{\mathrm{c}}$ is due to an increase of the texture quality and the Bi2223 content. The thermo-mechanical treatment allows the growth of $\mathrm{Bi} 2223$ crystallites on the Bi2212 ones and in the same time, the alignment of plate-like grains. The evaluation of the crystallite orientation distribution is then adequate to explain the evolution of physical properties.
A third graph in Fig. 3 presents our results on Bi2223 stacked-sinter forged Bi2223 discs. This study was developed in the view of high power applications, where the values of $I_{\mathrm{c}}$ should be as high as possible. To obtain a high $\mathrm{I}_{\mathrm{c}}$, several single discs were stacked thermomechanically under various uniaxial stresses. Fig. 3 shows the $I_{\mathrm{c}}$ and crystallite distribution values (FWHD) versus uniaxially applied stress during the heat treatment. One can note, (i) the dis-

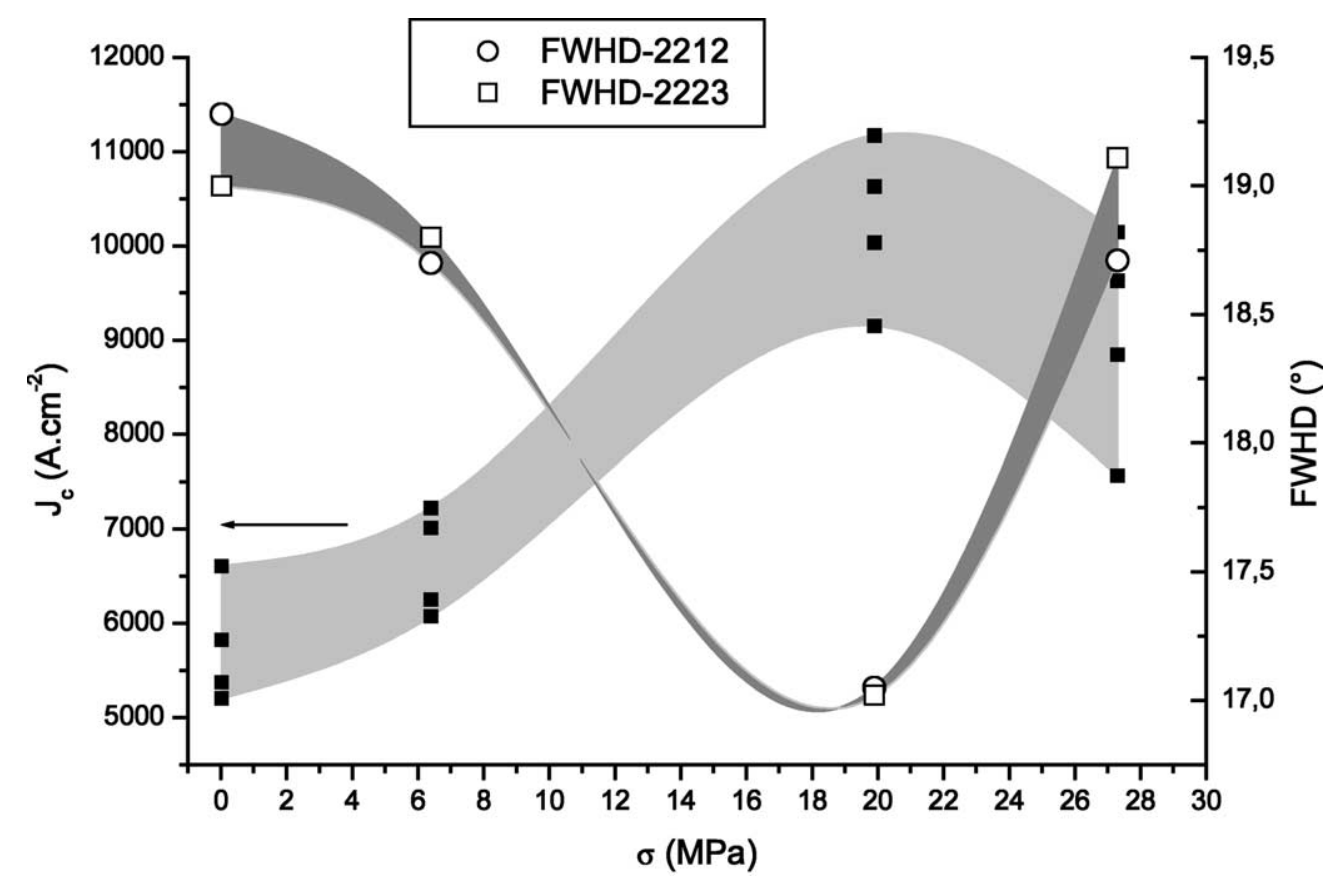

Fig. 3. Critical current density of hot-stacked samples showing the $J_{\mathrm{c}}$ 's-area and FWHDs versus applied stress [6]. 
persion of $I_{\mathrm{c}}$ from bar to bar cut in each sample (same applied stress), (ii) the $J_{\mathrm{c}}$ increase with increasing stresses up to a maximum around $20 \mathrm{MPa}$ and (iii) the correlated decrease of the FWHD of both superconducting phases with increasing the stress with a minimum also around $20 \mathrm{MPa}$. The inverse trend of the $J_{\mathrm{c}}$ and FWHD curves is quite interesting. It proves clearly that the transport properties are directly correlated to the texture quality of the forged discs down to FWHDs as low as $17^{\circ}$. The optimal value of applied stress to attain highest critical current densities corresponds also to the optimal one to induce better grain connectivity and alignment, i.e. lower FWHDs. In the process conditions, a reduction of the FWHD of around $2^{\circ}$ induces a $100 \% J_{\mathrm{c}}$ gain approximately for $20 \mathrm{MPa}$ of applied stress and for FWHDs in the $18^{\circ}$ range. It should be noticed that this effect is observed for closely developed textures between the Bi2212 and Bi2223 phases which ensures enough links for the current paths in the compound. These close textures are coming from the nucleation-growth mechanisms between the two phases, which is achieved in the sinter-forging method [4]. We do not have any hint at the moment whether if achieving FWHDs smaller than $14^{\circ}$ [5] may still enlarge $J_{\mathrm{c}}$ 's, but compared to YBCO thin film textural values [11] the ones observed in this work are 4-6 times larger.

\section{Combined texture/structure/particle size/phase ratio analysis of a $\mathrm{Y}-\mathrm{Ba}-\mathrm{Cu}-\mathrm{O}$ foam}

A foam constituted of the two phases $\mathrm{YBa}_{2} \mathrm{Cu}_{3} \mathrm{O}_{7-\delta}$ (YBCO123) and $\mathrm{Y}_{2} \mathrm{BaCu}_{5}$ (YBCO211), shown in Fig. 4, is an example of a sample very difficult to analyse due to its high porosity. Non-destructive $\mathrm{X}$-ray investigation is simply not possible on such a sample. But neutrons are able to probe its whole volume. We then used the combined approach on 1368 diagrams measured in as many sample orientations, from measurements performed at ILL on the D1B line as detailed elsewhere [12]. The relative amount of the two phases with their cell parameters and isotropic crystallite sizes were first refined on the sum diagram over all the sample orientation (Fig. 5), with satisfactory reliability factors $\left(R_{\mathrm{W}}=5.43 \%\right.$, $R_{\text {Bragg }}=19.71 \%$ ). A calcite sample with large crystallites and without deformation was used as a standard. We obtained $46(1) \%$ of YBCO123 in this sample, with no evidence of any other parasitic phase than YBCO211 (54(2)\% in volume). The cell parameters of the superconducting $(a=3.8128(6) \AA, b=3.8803(9) \AA, c=11.662(4) \AA)$ and insulating ( $a=12.158(4) \AA, b=5.645(2) \AA, c=7.117(3) \AA)$ phases are in good agreement with the abundant literature. The isotropic crystallite sizes are estimated to $136( \pm 32) \mathrm{nm}$ and $139( \pm 43) \mathrm{nm}$ respectively for the two phases, without high precision due to the relatively low resolution of the instrument.

In a second step, the ODF was extracted using the WIMV formalism [13] implemented in MAUD [9], other parameters being fixed. The OD was then used to recalculate the (003) pole figure (Fig. 6). This latter shows level of orientation as high as around 100 times the random powder. The sample exhibits four main orientation components with widths at half-maxima of $10^{\circ}$ approximately, and orientations close to the main axes of the sample. Domains A, B and $\mathrm{C}$ have $c$-axes at around $25^{\circ}$ from the $X$ and $Y$ axes respectively, while domain $\mathrm{D}$ has its $c$-axes at no more than $20^{\circ}$ from $\mathrm{Z}$. The texture development in this sample is very similar to the melt grown samples of such phases, whereas a priori no texture could be supposed from the extremely complex shapes of the alveoli of the foam. This texture stabilisation explains the relatively good superconducting properties of the sample $\left(T_{\mathrm{c}}=91 \mathrm{~K}, J_{\mathrm{c}}=40000 \mathrm{~A} \mathrm{~cm}^{-2}\right)$ [14].
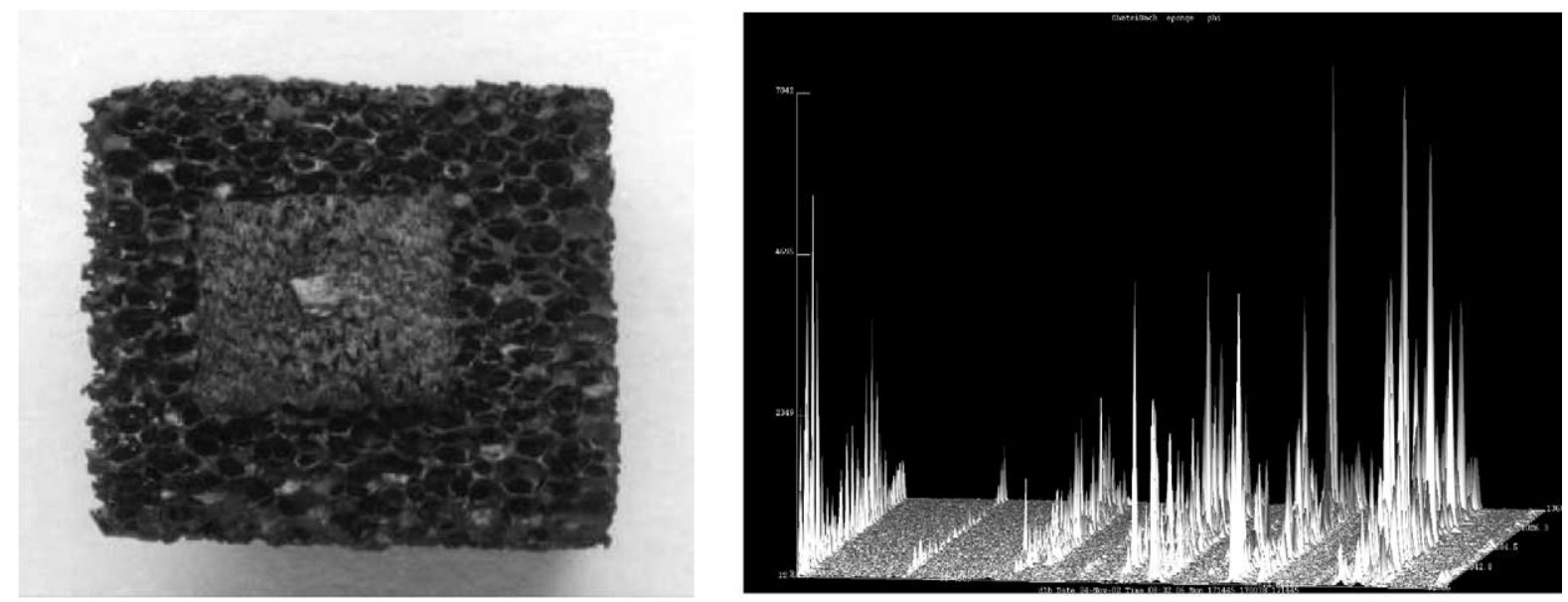

Fig. 4. Textured foam (left), as synthesized and as analysed using neutrons (right). Note the strong variations of the peak intensities when rotating the sample, indicative of a strong texture in the sample. 


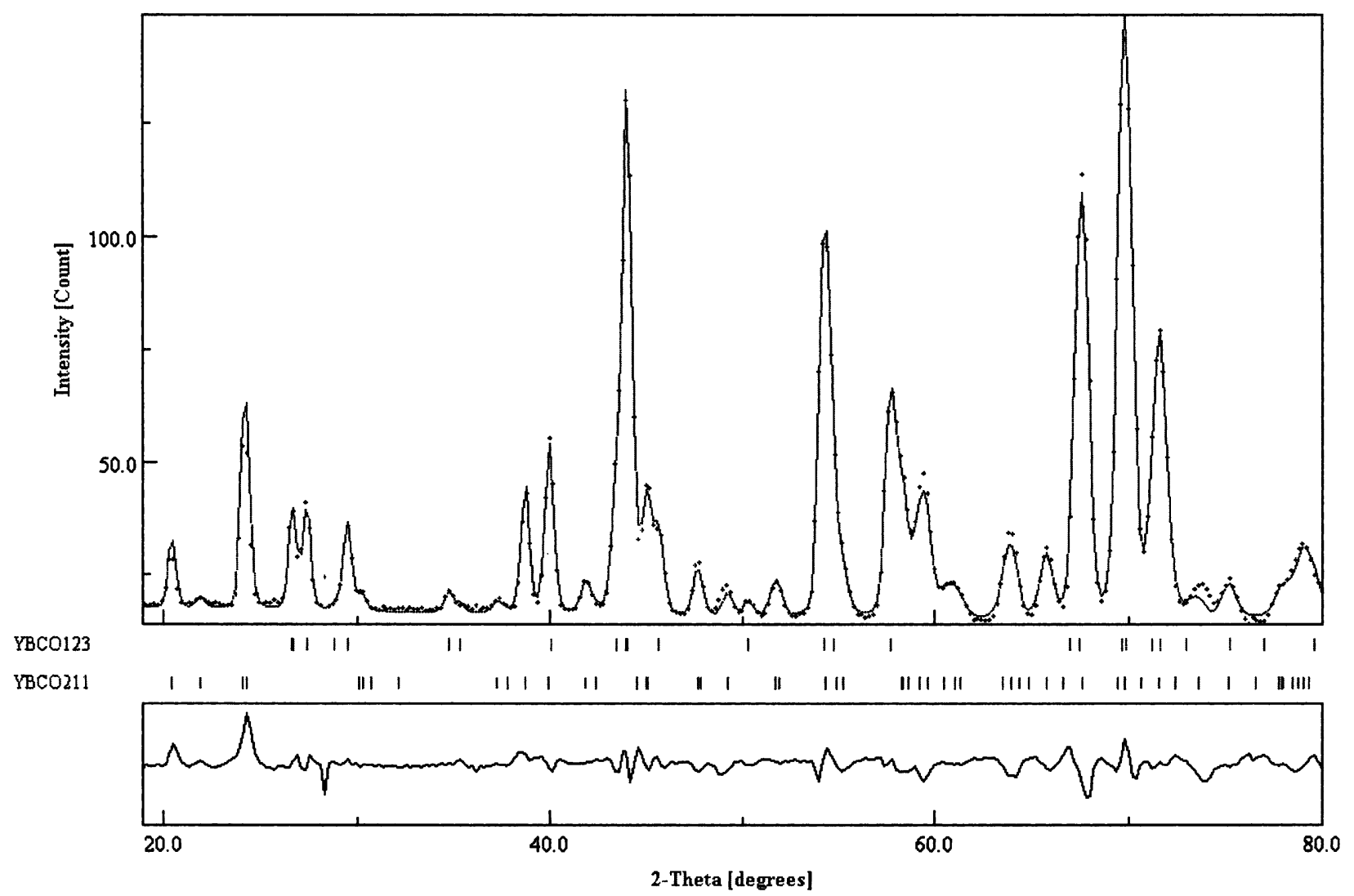

Fig. 5. Rietveld refinement of the sum of the 1368 measured diagrams, allowing phase, particle size and cell parameters quantitative determinations. We obtained the following reliability factors: $R_{\mathrm{W}}=5.43 \%, R_{\mathrm{Bragg}}=19.71 \%$.

These first observations are very encouraging in the view of obtaining single domains in such bulk samples with physical properties comparable to the best ones measured in $\mathrm{Y}-\mathrm{Ba}-\mathrm{Cu}-\mathrm{O}$ oriented compounds.

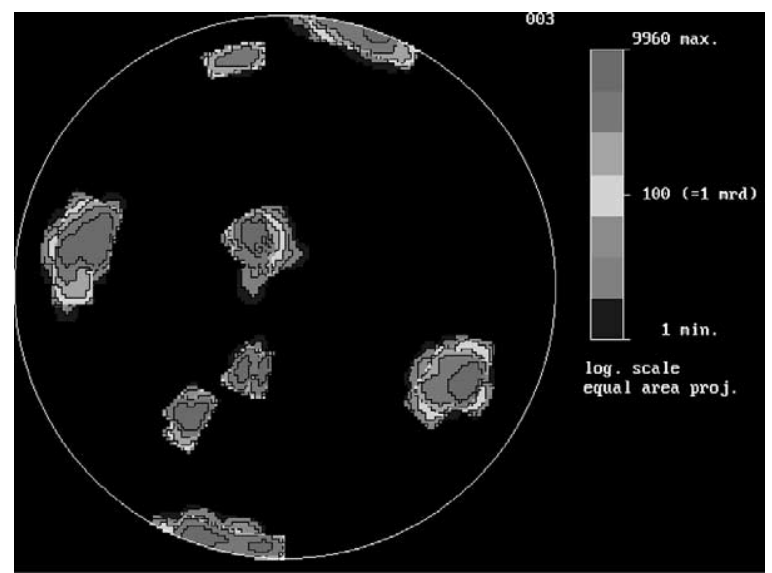

Fig. 6. (003) pole figure showing the very strong orientation of the YBCO123 phase, with four main domains.

\section{Conclusion}

A correlation between texture stabilisation and physical properties has been established in Bi2223 samples. It reveals the importance of a precise texture development in our materials. The crystallite orientation distribution sharpness is generally a key point to explain the superconducting properties of Bi2223 textured discs. Neutron diffraction measurements on Y123 foams present promising results for the future using the combined analysis.

\section{References}

[1] F.K. Lotgering, J. Inorg. Nucl. Chem. 9 (1959) 113.

[2] M. Field, M.E. Merchant, J. Appl. Phys. 20 (1949) 741.

[3] H.-J. Bunge, C. Esling, Quantitative Texture Analysis, Bunge and Esling Ed., DGM, Germany, 1982.

[4] E. Guilmeau, D. Chateigner, J.G. Noudem, Supercond. Sci. Technol. 15 (2002) 1436

[5] E. Guilmeau, D. Chateigner, J.G. Noudem, Supercond. Sci. Technol. 16 (2003) 484.

[6] J.G. Noudem, E. Guilmeau, D. Chateigner, Physica C, in press.

[7] H.-R. Wenk, D. Chateigner, M. Pernet, J. Bingert, E. Hellstrom, B. Ouladdiaf, Physica C 272 (1996) 1. 
[8] J. Ricote, D. Chateigner, Boletín de la Sociedad Española de Cerámica y Vidrio 38 (6) (1999) 587.

[9] L. Lutterotti, S. Matthies, H.-R. Wenk, in: J.A. Szpunar (Ed.), Textures of Materials , vol. 2, NRC Research Press, Ottawa, 1999, 1599

[10] E. Guilmeau, J.G. Noudem, Supercond. Sci. Technol. 15 (2002) 1566.

[11] M. Pernet, D. Chateigner, P. Germi, C. Dubourdieu, O. Thomas, J.P. Sénateur, D. Chambonnet, C. Belouet, Physica C 235-240 (1994) 627.
[12] D. Chateigner, H.-R. Wenk, M. Pernet, J. Appl. Crystallogr. 30 (1997) 43.

[13] S. Matthies, G.W. Vinel, Phys. Status Solidi B 112 (1982) 111

[14] J.G. Noudem, E. Guilmeau, S. Lambert, E.S. Reddy, B. Ouladdiaf, G.J. Schmitz, M.S. Rio, Physica C, in press. 